\title{
Delivery of vincristine sulfate-conjugated gold nanoparticles using liposomes: a light-responsive nanocarrier with enhanced antitumor efficiency
}

This article was published in the following Dove Press journal:

International Journal of Nanomedicine

22 April 2015

Number of times this article has been viewed

Ying Liul,*

Man $\mathrm{He}^{1, *}$

Mengmeng Niu'

Yiqing Zhao'

Yuanzhang Zhu'

Zhenhua $\mathrm{Li}^{2}$

Nianping Feng ${ }^{1}$

'Department of Pharmaceutical Sciences, School of Pharmacy,

Shanghai University of Traditional

Chinese Medicine, Shanghai, People's

Republic of China; ${ }^{2}$ Cedars-Sinai

Medical Center, Los Angeles, CA, USA

*These authors contributed equally to this work
Correspondence: Nianping Feng

Department of Pharmaceutical Sciences, School of Pharmacy, Shanghai University of Traditional Chinese Medicine, 1200

Cailun Road, Zhangjiang Hi-Tech Park,

Pudong New District, Shanghai 201203,

People's Republic of China

Tel +862151322198

$\mathrm{Fax}+862151322198$

Email npfeng@hotmail.com;

npfeng@shutcm.edu.cn
Abstract: Rapid drug release at the specific site of action is still a challenge for antitumor therapy. Development of stimuli-responsive hybrid nanocarriers provides a promising strategy to enhance therapeutic effects by combining the unique features of each component. The present study explored the use of drug-gold nanoparticle conjugates incorporated into liposomes to enhance antitumor efficiency. A model drug, vincristine sulfate, was physically conjugated with gold nanoparticles and verified by UV-visible and fourier transform infrared spectroscopy, and differential scanning calorimetry. The conjugates were incorporated into liposomes by film dispersion to yield nanoparticles $(113.4 \mathrm{~nm}$ ) with light-responsive release properties, as shown by in vitro release studies. Intracellular uptake and distribution was studied in HeLa cells using transmission electron microscopy and confocal laser scanning microscopy. This demonstrated liposome internalization and localization in endosomal-lysosomal vesicles. Fluorescence intensity increased in cells exposed to UV light, indicating that this stimulated intracellular drug release; this finding was confirmed by quantitative analyses using flow cytometry. Antitumor efficacy was evaluated in HeLa cells, both in culture and in implants in vivo in nude mice. HeLa cell viability assays showed that light exposure enhanced liposome cytotoxicity and induction of apoptosis. Furthermore, treatment with the prepared liposomes coupled with UV light exposure produced greater antitumor effects in nude mice and reduced side effects, as compared with free vincristine sulfate.

Keywords: vincristine sulfate, vincristine sulfate-gold nanoparticle conjugates, liposomes, antitumor efficiency, light triggered drug release

\section{Introduction}

Impressive progress has been made in the diagnosis and treatment of cancer during the past decades. Various nanocarriers have been designed to deliver these chemotherapeutic agents, and liposomes, polymeric nanoparticles, micelles, and dendrimers have shown promising results. ${ }^{1}$ However, controlled drug delivery, with rapid drug release and accumulation at the tumor site, is still a major challenge for efficient therapy of cancers.

To address these concerns, various stimuli-responsive nanocarriers have been explored in recent years. ${ }^{2}$ Drug release can be triggered by various features of the tumor microenvironment, such as $\mathrm{pH}$ or temperature ${ }^{3-5}$ alternatively, the unique properties of inorganic nanocarriers can be exploited to trigger drug release in response to a range of external stimuli including light, ultrasound, and magnetic or electrical fields. Light activation is relatively easy to apply to anticancer therapy. Gold nanoparticles have the unique features of strong light absorption and distinct surface plasmon resonance, and 
can convert light into heat. They have good biocompatibility and stability, and almost no toxicity. ${ }^{6}$ Surface modification allows gold nanoparticle to be conjugated with many therapeutic agents including oligonucleotides, proteins, and chemotherapeutic agents. ${ }^{7}$ The conjugates can be delivered to cells where they accumulate in intracellular compartments, ${ }^{8}$ and suppress multidrug resistance (MDR). ${ }^{9}$ Controlled and site-specific drug release can be triggered by internal stimuli, such as glutathione level and $\mathrm{pH}$, or by an external stimulus, such as light. ${ }^{10-12}$ Conversion of the absorbed light into heat facilitates tumor cell killing. ${ }^{13,14}$ Furthermore, gold nanoparticles have been used to enhance the effects of radiation in cancer treatment. ${ }^{15}$ Therefore, these features enhanced their potential for cancer therapy. ${ }^{16,17}$

However, their application in antitumor therapy is hindered by their low targeting capacity, short circulation half-lives, and their rapid entrapment and clearance by the reticuloendothelial system (RES). ${ }^{15,18}$ Liposomes are one of the most promising drug carriers for cancer treatment, both in preclinical development and clinical application, and they show a number of advantages including good biological compatibility, reduced toxicity, and improved drug stability. Incorporation into liposomes may provide a viable strategy to address these problems, in which liposomes could increase tumor delivery of the gold nanoparticles, reduce their RES entrapment, and reduce side effects; while the gold nanoparticles would increase the intracellular drug concentration and enable light-triggered drug release.

To date, most of the research relating to liposomes and gold nanoparticles has not investigated drug loading ${ }^{17,19-21}$ or has separately incorporated gold nanoparticles and free drug into liposomes. ${ }^{22-24}$ Therefore, the present study investigated a light-responsive delivery system by incorporating druggold nanoparticle conjugates into liposomes, to enhance antitumor efficacy and reduce toxicity. The model drug, vincristine sulfate (VCR) is a water-soluble alkaloid derived from Catharanthus roseus. VCR is widely used clinically to treat a range of cancers including acute lymphoblastic leukemia, cervical cancer, and breast cancer. The mechanism involves microtubule inhibition and arrest of cell division in metaphase. ${ }^{25}$ However, severe side effects such as dosedependent neurotoxicity and MDR have limited its clinical application. ${ }^{26}$ In the present study, VCR was electrostatically bound to gold nanoparticle, followed by incorporating into liposomes. The light-triggered release and intracellular delivery were evaluated quantitatively and qualitatively in HeLa cells. Light-responsive antitumor efficacy was evaluated in both HeLa cells and in tumor-bearing nude mice. The in vivo biodistribution of the prepared liposomes and their induction of tumor cell apoptosis were also investigated.

\section{Materials and methods Materials}

VCR was obtained from Nanjing Zelang Medical Technology Co. Ltd., Jiangsu, People's Republic of China. Hydrogen tetrachloroaurate hydrate $\left(\mathrm{HAuCl}_{4} \cdot 3 \mathrm{H}_{2} \mathrm{O}\right)$ was purchased from the Aladdin-chemistry Co. Ltd. (Shanghai, People's Republic of China). Sodium citrate $\left[\mathrm{HOC}(\mathrm{COONa})\left(\mathrm{CH}_{2} \mathrm{COONa}\right)_{2} \cdot 2 \mathrm{H}_{2} \mathrm{O}\right]$ and dimethylsulfoxide (DMSO) were purchased from Sinopharm Group Co. Ltd. (Shanghai, People's Republic of China). Egg yolk lecithin (EYPC; PC-98T, purity >98\%), 1,2-distearoyl-sn-glycero-3-phosphoethanolamine- $N$ methoxy(polyethyleneglycol)-2000 (DSPE-PEG2000), and cholesterol were provided by Q.P. corporation (Tokyo, Japan). All other chemicals were of high-performance liquid chromatography (HPLC) or analytical grade. Dulbecco's Modified Eagle's Medium (DMEM, high glucose), fetal bovine serum (FBS, Australian origin), penicillin and streptomycin, $1 \times$ trypsin-ethylenediaminetetraacetic acid (EDTA) solution ( $0.25 \%$ trypsin with $0.53 \mathrm{mM}$ EDTA), and Lyso-Tracker Red (DND-99) were from Life Technologies (Grand Island, NY, USA); 3-(4,5-dimethylthiazol-2yl)-2,5, diphenyltetrazolium bromide (MTT reagent), Hoechst 33258, calcein, and rhodamine B were from Sigma Aldrich (St Louis, MO, USA). Purified deionized water (Millipore, Bedford, MA, USA) was used in all experiments.

HeLa cells were obtained from the Institute of Biochemistry and Cell Biology (Shanghai, People's Republic of China). The cells were cultured in DMEM containing 10\% FBS, penicillin (100 IU/mL), and streptomycin $(100 \mu \mathrm{g} / \mathrm{mL})$ at $37^{\circ} \mathrm{C}$ in the presence of $5 \% \mathrm{CO}_{2}$.

Female BALB/c nude mice ( $20 \pm 2 \mathrm{~g}$ ) were obtained from Shanghai SLAC Laboratory Animal Ltd. (Shanghai, People's Republic of China). The animals were bred and maintained at $25^{\circ} \mathrm{C}$ with a relative humidity of $55 \%$, under standard conditions with free access to food and water (Shanghai University of TCM, Shanghai, People's Republic of China). The animal care and experiments were performed with the approval of the institutional animal ethical committee of the Shanghai University of TCM.

\section{Preparation and characterization of VCR-gold nanoparticle conjugates (VGC)}

Gold nanoparticles were prepared using the Turkevich-Frens method. ${ }^{27}$ Briefly, $150 \mathrm{~mL} \mathrm{HAuCl}{ }_{4}$ solution $\left(2.7 \times 10^{-3} \mathrm{M}\right)$ was heated to boiling in a dimethicone oil bath, and $9 \mathrm{~mL}$ of 
$0.3 \mathrm{M}$ trisodium citrate was quickly added to the boiled $\mathrm{HAuCl}_{4}$ solution. Then, the mixture was continuously stirred for 12 minutes. Finally, the mixture was cooled to room temperature, and kept at $4^{\circ} \mathrm{C}$ prior to further use.

The resulting gold nanoparticles were conjugated with VCR solution at a molar ratio of 6:100. In brief, VCR solution was slowly added to the gold nanoparticle dispersion. The mixture was then stirred for 2 hours at room temperature, followed by centrifugation at $12,000 \mathrm{rpm}$ for 10 minutes to remove free VCR. The sediment (VGC) was resuspended in deionized water.

The synthesized gold nanoparticles and VGC were characterized by analyses of particle size and zeta potential using Zetasizer nano-ZS 90 (Malvern Instruments, Malvern, UK), imaging using transmission electron microscopy (TEM, JEOL-1230 transmission electron microscope, JEOL Ltd., Tokyo, Japan), and UV-visible spectrophotometry (INESA INSTRUMENT Ltd., Shanghai, People's Republic of China). For TEM imaging, gold nanoparticles or VGC were dispersed in deionized water and dropped onto 200- to 400-mesh copper grids, followed by removal of excess liquid using filter paper. After air-drying at room temperature, the specimens were observed and photographed using a TEM at an acceleration voltage of $200 \mathrm{kV}$. The obtained VGC were further characterized by fourier transform infrared spectroscopy (FTIR) and differential scanning calorimetry (DSC) analyses to determine the physical state of the drug molecule present in the conjugate and to confirm conjugate formation. FTIR was measured using an AVATAR 330 FTIR spectrometer (Nicolet, Waltham, MA, USA), scanning from 400 to $4,000 \mathrm{~cm}^{-1}$ at $4 \mathrm{~cm}^{-1}$ resolution. DSC spectra were obtained using NETZSCH DSC 204 apparatus (NETZSCH Gerätebau $\mathrm{GmbH}$, Selb, Germany) with a scan rate of $10^{\circ} \mathrm{C}$ per minute at $30^{\circ} \mathrm{C}-250^{\circ} \mathrm{C}$ under a nitrogen purge.

\section{Preparation and characterization of VGC-loaded liposomes (VGC-L)}

Liposomes were prepared by the thin film hydration method, as previously described. ${ }^{28}$ In brief, lipids (EYPC:DSPEPEG2000, 90:10, w/w) were dissolved in an organic solution of trichloromethane:methanol $(3: 1, \mathrm{v} / \mathrm{v})$. After removal of the organic solvent under reduced pressure, a lipid film was obtained. It was hydrated with the VGC (prepared above) for 1 hour. Finally, the resulting VGC-L dispersions were sonicated at $100 \mathrm{~W}$ for 5 minutes.

Calcein and rhodamine $\mathrm{B}$ were used as hydrophilic fluorescent markers to probe the cellular uptake and in vivo distribution of liposomes, respectively. Gold nanoparticles conjugated to these fluorescent markers and conjugate-loaded liposomes were prepared as described above, replacing VCR with the fluorescent markers.

The encapsulation efficiency (EE) of VGC-L was determined by ultrafiltration. The VGC-L were introduced into centrifugal filter tubes (molecular weight cut-off [MWCO]: $300 \mathrm{kDa}$, Bedford, MA, USA) and centrifuged at 5,000 rpm for 20 minutes. The VCR concentration in the liposomes was measured by HPLC using an Agilent 1200 series liquid chromatography system (Agilent Technologies, Santa Clara, CA, USA), equipped with a reverse-phase C18 column (Diamonsil C18, 250×4.6 mm, $5 \mu \mathrm{m}$ ). The injection volume was $20 \mu \mathrm{L}$ and the mobile phase consisted of methanol and deionized water $(75: 25, \mathrm{v} / \mathrm{v})$ adjusted to $\mathrm{pH} 7.0$ with $0.016 \%$ phosphoricacid, at a flow rate of $1 \mathrm{~mL} / \mathrm{min}$, with a detection wavelength of $297 \mathrm{~nm}$. The EE was calculated according to the following equation: $\mathrm{EE}(\%)=(\mathrm{VCR}$ amount in VGC-L/total amount added to the formulation) $\times 100 \%$.

\section{In vitro release}

After loading the VGC-L into a dialysis bag (MWCO, 8-10 $\mathrm{kDa}$ ), the in vitro release profile was determined in a release medium of phosphate-buffered saline (PBS), $\mathrm{pH} 7.4$, at $37^{\circ} \mathrm{C}$ under constant stirring. At predetermined times, $1 \mathrm{~mL}$ of release medium was withdrawn and replaced by fresh release medium. The content was analyzed using HPLC.

To determine the drug release behavior in acidic conditions, this in vitro release study was also carried out in acetate buffer release medium ( $\mathrm{pH} 4.5$ ). Furthermore, light-induced release was evaluated by illumination with UV light at $365 \mathrm{~nm}$.

\section{Cell uptake}

Cell uptake and intracellular distribution were studied to evaluate the intracellular delivery of the VGC-L. HeLa cells were seeded in 6-well plates at a density of $1 \times 10^{5}$ cells per well. After incubation for 24 hours, the culture medium was replaced with fresh medium containing VGC-L. The plates were incubated at $37^{\circ} \mathrm{C}$ for 2 hours. To determine the influence of light on cell uptake, the cells were illuminated with UV light (40W, LP-40, Shanghai Luyang Equipment Co., Ltd, China) for 120 seconds after 1 hour incubation, and then continually incubated for 1 hour. The cells were washed with PBS three times, fixed with $2.5 \%$ glutaraldehyde, scraped, and collected. The cells were then washed with PBS, treated with osmic acid-phosphate buffer for 2 hours, serially dehydrated using ethanol and acetone 
for 1 hour, embedded in resin for 12 hours, and cut into thin sections using an ultramicrotome. The ultrathin sections were collected on 200-400 mesh grids, stained with 1\% uranyl acetate for 30 minutes and lead citrate for 10 minutes, and washed in $0.02 \mathrm{M} \mathrm{NaOH}$ solution, followed by deionized water. After removing excess liquid using filter paper, the sections were imaged using TEM (JEOL-1230, JEOL Ltd.) at an operating voltage of $200 \mathrm{kV}$.

To investigate cell uptake of fluorescently-labeled liposomes, HeLa cells were seeded in glass-bottomed Petri dishes at a density of $1 \times 10^{5}$ cells per well. After incubation for 24 hours, fresh medium containing liposomes loaded with calcein-conjugated gold nanoparticles (CGC-L) was added and incubated for 30, 60, and 120 minutes. Some of these cells were exposed to UV light illumination for 120 seconds, 10 minutes prior to end of the incubation period. After incubation, the cells were washed with PBS, fixed in $4 \%$ paraformaldehyde at $4{ }^{\circ} \mathrm{C}$ for 30 minutes, washed with TPBS (PBS containing $0.4 \%$ Tween-20), and stained with Hoechst $33258(10 \mu \mathrm{g} / \mathrm{mL})$ for 30 minutes. The cells were then washed with TPBS, stained with LysoTracker Red DND 99 (400 nM) for 30 minutes, and washed with TPBS. The cells were observed using confocal laser scanning microscopy (CLSM) at excitation wavelengths of 415, 498, and $522 \mathrm{~nm}$ for Hoechst 33258 (blue), calcein (green), and Lyso-Tracker Red (red), respectively.

For flow cytometry, HeLa cells were seeded into 6-well culture plates $\left(1 \times 10^{5}\right.$ cells per well). After incubation for 24 hours, culture medium containing CGC-L was added to the wells and cultured for 1 hour. Some of the cells were exposed to UV light illumination for 120 seconds and then further cultured for 1 hour. After washing with PBS ( $\mathrm{pH} 7.4$ ) three times, the cells were collected and kept in PBS prior to flow cytometry analysis. Cells cultured in blank culture medium were used as a negative control.

\section{Cytotoxicity assay}

HeLa cells were seeded in 96-well plates and incubated for 24 hours prior to adding culture medium containing VGC-L to the well and then cultured for 4 hours at $37^{\circ} \mathrm{C}$. The cells were then exposed to UV light for 60,120 , or 180 seconds. The cells were further incubated for 20 hours, and MTT assay was used to evaluate the effect of VGC-L on cell viability. Cells exposed to free VCR or gold nanoparticle-free VCR-loaded liposomes (VCR-L) were used as controls.

Flow cytometry was used to evaluate the influence of VGC-L on cell apoptosis. Cells were seeded in 6-well plates at a density of $1 \times 10^{5}$ cells per well. After incubation for 24 hours, the blank culture medium, VCR-L, or VGC-L were incubated with cells for 1 hour. Some cells were then exposed to UV light for 120 seconds, and subsequently cultured for 1 hour. The cells were washed with PBS three times, collected, and fixed in ethanol (70\%). Then, the cells were washed with PBS three times, followed by incubation in PBS for 0.5 hours. Cells that were not illuminated with UV light were used as the control.

Optical microscopy was used to examine the effects of liposomes on cell viability. Cells were treated as described above, except that after exposure to UV light for 120 seconds, cells were incubated for 1 or 2 hours prior to washing with PBS and examined using an optical microscope. Naïve HeLa cells (no treatment) were used as the control.

\section{In vivo antitumor activity}

HeLa cells $\left(2 \times 10^{6}\right.$ cells $)$ were harvested and subcutaneously injected into the armpit of BALB/c nude mice to establish a tumor model. When the tumor volume reached approximately $150 \mathrm{~mm}^{3}$, the mice were randomly divided into five groups (five animals per group) as follows: saline negative control group, UV laser only group (without any drug or preparation treatment), VCR group, VGC-L group, and VGC-L + UV laser group. The test samples were administered by IV injection into the tail vein every 2 days at a dosage of $0.6 \mathrm{mg} / \mathrm{kg}$. For the VGC-L + UV laser group, mice were exposed to UV light for 120 seconds, 2 hours after each intravenous (IV) administration. The tumor volume $(V)$ and body weight were measured every 2 days. Tumor volume $(V)$ was calculated using the following equation: $V$ $=0.5 \times$ length $\times$ width $^{2}$.

The animals were sacrificed on day 15 and the tumors were excised, weighed, and photographed. The tumor inhibitory rate (TIR) was calculated as follows: TIR = (tumor weight of control group - tumor weight of treated group)/tumor weight of control group $\times 100 \%$. In addition, an analysis of cell apoptosis in tumor sections was performed by Terminal-deoxynucleoitidyl Transferase Mediated Nick End Labeling (TUNEL) assay using an in situ cell death detection kit (Roche Diagnostics GmbH, Germany) according to the manufacturer's instructions.

To investigate the in vivo distribution of the prepared liposomes, in vivo imaging was performed. Rhodamine B/ gold nanoparticle-loaded liposomes (RGC-L) were administered via IV injection to the tumor-bearing mice, and stimulated by exposure to UV light.

\section{Statistical analysis}

Data are presented as the mean \pm standard deviation. Statistical analyses were performed using one-way analysis of variance 
(ANOVA). A $P$-value of $<0.05$ was considered statistically significant.

\section{Results}

\section{Preparation and characterization of VGC}

Gold nanoparticles were synthesized by reducing tetrachloroaurate, using trisodium citrate as the reducing agent. The mean size of the resultant citrate-capped gold nanoparticles was $16 \mathrm{~nm}$. The color changed from pale yellow to ruby red upon formation of the gold nanoparticles, consistent with the literature. ${ }^{29}$ Investigation of gold nanoparticle morphology (Figure 1C) revealed homogeneous, dispersed, spherical particles with a smooth surface (when observed by TEM) and a nanosize that was consistent with the dynamic light scattering (DLS) results. UV-visible spectroscopy found a maximum UV absorption wavelength at $519 \mathrm{~nm}$ (Figure 1B) due to the surface plasmon resonance absorption of the gold nanoparticles. The zeta potential of the prepared gold nanoparticles was $-40.6 \mathrm{mV}$. These results indicated that the citrate-capped gold nanoparticles had been prepared successfully.

Various VCR:gold nanoparticle ratios (3:100 to 8:100) were explored and the resulting VGC particle size, zeta potential, conjugation efficiency, and drug loading are shown in Figure 1A. VCR conjugation with the gold nanoparticles reached almost $100 \%$ at ratios below 6:100, while the conjugation rates decreased significantly at 8:100; drug loading efficiency was not significantly different at the 6:100 and 8:100 ratios. The highest conjugation efficiency was achieved at the ratio of $6: 100$. Below this ratio, unconjugated

\begin{tabular}{clllll}
\hline A Sample & Zeta potential $(\mathbf{m V})$ & Particle size $(\mathbf{n m})$ & PDI & EE (\%) & DL $(\%)$ \\
\hline VCR:gold nanoparticles $(\mathrm{mol}: \mathrm{mol})$ & & & & \\
8:100 & $29.6 \pm 2.2$ & $38.8 \pm 4.2$ & $0.42 \mathrm{I} \pm 0.05 \mathrm{I}$ & $77.0 \pm 3.0$ & $21.3 \pm 0.66$ \\
6:100 & $-28 \pm 1.4$ & $23.8 \pm 2.4$ & $0.455 \pm 0.034$ & $99.2 \pm 0.1$ & $20.8 \pm 0.0 \mathrm{I}$ \\
3:100 & $-23 \pm 2.7$ & $18.5 \pm 1.3$ & $0.445 \pm 0.042$ & $105.5 \pm 2.9$ & $15.7 \pm 0.37$ \\
Gold nanoparticles & $-40.6 \pm 3.5$ & $17.7 \pm 4.6$ & $0.436 \pm 0.062$ & - & - \\
Blank liposomes & $-2.4 \pm 1.2$ & $92.3 \pm 0.9$ & $0.325 \pm 0.055$ & - & - \\
VGC-L & $-11.3 \pm 2.2$ & $113.4 \pm 1.7$ & $0.289 \pm 0.009$ & $75.4 \pm 0.2$ & $15.9 \pm 0.1$ \\
\hline
\end{tabular}

B VCR:gold nanoparticles (mol:mol)

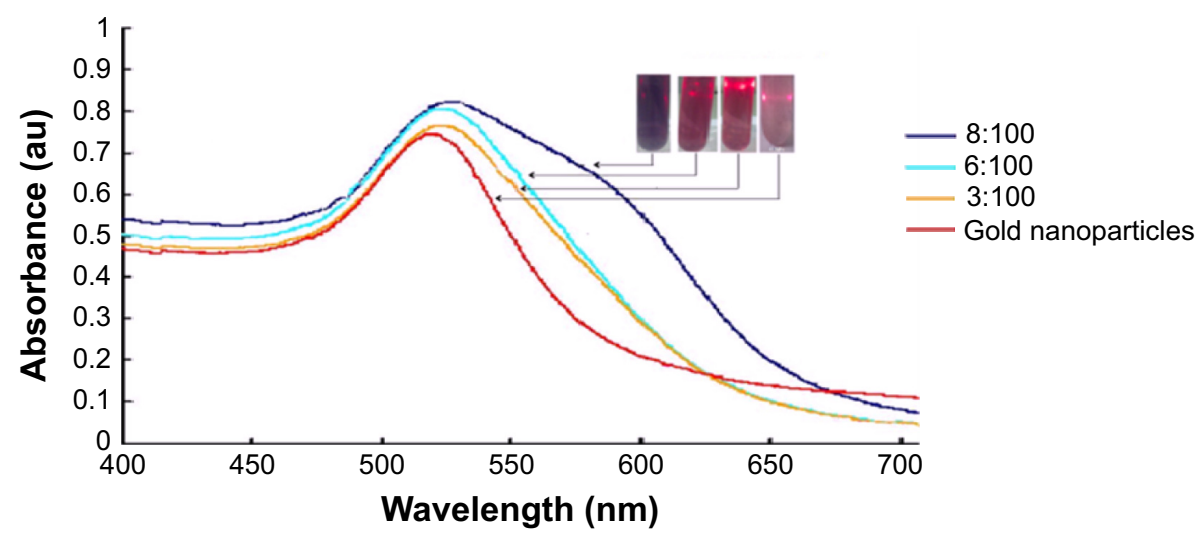

C

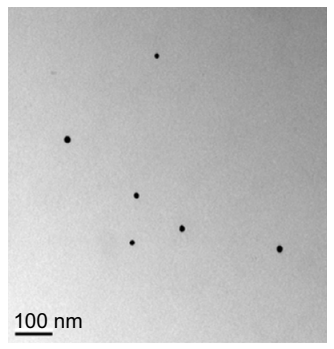

D

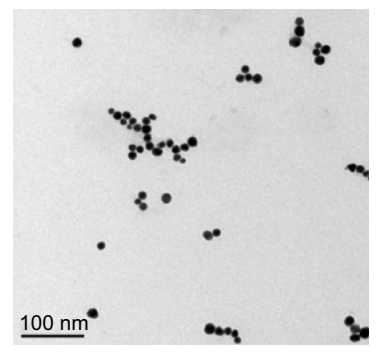

E

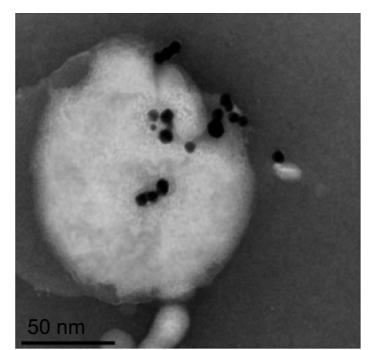

Figure I Characterization of gold nanoparticles, VGC, and VGC-L.

Notes: (A) Particle size, zeta potential, encapsulation efficiency, and drug loading ( $n=3)$; (B) UV-visible spectra; (C) TEM images of gold nanoparticles $(\times 100,000$ magnification); (D) TEM images of VGC ( $\times 100,000$ magnification); (E) TEM images of VGC-L ( $\times 100,000$ magnification).

Abbreviations: PDI, polydispersity index; EE, Encapsulation efficiency; DL, drug loading; VGC-L, vincristine sulfate-gold nanoparticles conjugates-loaded liposomes; TEM, transmission electron microscopy; VGC, vincristine sulfate-gold nanoparticles conjugates; VCR, vincristine sulfate. 
gold nanoparticles were present, while above this ratio, free VCR was present. The formation of VGC was confirmed by UV-visible spectroscopy (Figure 1B). When the ratio of VCR increased, a red shift was observed (increase in wavelength) which implied an increase in particle size, consistent with the DLS determination. In addition, an obvious increase in the intensity of the absorption peak signified an increased VGC concentration. In accordance with the results described above, a VCR:gold nanoparticle ratio of 6:100 was selected to prepare the VGC used in the subsequent studies.

TEM imaging showed that the VGC particles were spherical and uniform (Figure 1D). The FTIR patterns for VCR, gold nanoparticles, and VGC are shown in Figure S1A. The characteristic peak of VCR at $1,669 \mathrm{~cm}^{-1}$ was shifted to $1,634 \mathrm{~cm}^{-1}$ in VGC, suggesting the interaction between VCR and gold nanoparticles occurred in VGC. In addition, VCR showed a characteristic peak at $227.2^{\circ} \mathrm{C}$ on the DSC curve (Figure S1B) and this peak disappeared in VGC, indicating a change in the crystal form.

The stability of VGC was investigated using UV-visible spectroscopy to identify aggregation or other forms of instability under a range of $\mathrm{pH}$ and ionic strength conditions. As shown in Figure 2A, the UV-visible spectra were almost unchanged at $\mathrm{pH}$ values larger than 5, indicating good stability under these conditions. Decreasing the $\mathrm{pH}$ to below 5 resulted in a significant red shift, with a maximum UV-absorption wavelength from 520 to $680 \mathrm{~nm}$, and the appearance of two broad peaks, suggesting a change in particle size and/or shape. ${ }^{30}$ Furthermore, the UV-visible spectral intensity decreased as the $\mathrm{pH}$ dropped below 5 and the $520 \mathrm{~nm}$ peak flattened and almost disappeared, indicating a reduced VGC concentration. Increasing the ionic strength also decreased VGC stability (Figure S1B). These findings indicated that the conjugation state of VGC may be compromised under acidic and high ionic strength conditions, liberating VCR.

The in vitro release study showed an accumulated release of $31 \%$ in PBS at $\mathrm{pH} 7.4$, as compared with $94 \%$ in acetate buffer, which was used to simulate the intracellular endosomallysosomal environment in tumor cells (Figure 2C). This feature met the requirement for efficient tumor treatment by rapidly releasing drugs within cancer cells, achieving a high concentration and enhancing antitumor efficiency. At the same time, these data suggested that release would be slower in other body fluids, reducing the toxicity to normal tissues and organs.

\section{Preparation and characterization of VGC-L}

VGC-L were prepared by thin-film dispersion. The results are shown in Figure 1A. The zeta potential of the blank liposomes changed from almost neutral $(-2.41 \mathrm{mV})$ to negative $(-11.3 \mathrm{mV})$ after $\mathrm{VGC}$ addition, indicating the formation of VGC-L. The polydispersity index (PDI) was $<0.3$, indicating a relatively uniform size distribution. TEM image (Figure 1E) showed almost round particles with a size consistent with the DLS results of $113 \mathrm{~nm}$. In addition, strong black dots of VGC were clearly visible, either encapsulated in the inner cavity or adsorbed to the surface of the VGC-L.

To investigate the state of VGC within liposomes, DSC was performed. VGC had a characteristic peak at $216.6^{\circ} \mathrm{C}$, which was shifted to $210^{\circ} \mathrm{C}$ in VGC-L and reduced in intensity (Figure S1C). In addition, the VGC peak at $66.7^{\circ} \mathrm{C}$ disappeared in VGC-L, revealing that VGC was in a less crystalline state in these liposomes.

\section{Controlled release under UV light}

Controlled, light-activated drug release is advantageous for antitumor therapy. Prior to investigating the effects of light on VGC-L drug release, in vitro release at different $\mathrm{pH}$ values was studied, as this can provide important insights into the in vivo activities of drug delivery systems. As shown in Figure $3 \mathrm{~A}, \mathrm{VCR}$ cumulative release from VGC-L at 8 hours was $6.3 \%$ and $13 \%$ at $\mathrm{pH} 7.4$ and 4.5 , respectively, and no significant burst release occurred under either $\mathrm{pH}$ condition, suggesting that VGC was incorporated into the aqueous liposome core, consistent with previous reports. ${ }^{31}$ In addition, drug release was accelerated by UV light exposure (Figure 3B).

\section{Intracellular uptake}

The uptake and localization of VGC-L in HeLa cells were qualitatively assessed by TEM and CLSM and quantitatively assessed by flow cytometry. In the CLSM and flow cytometry studies, calcein (a hydrophilic fluorescent molecule) was conjugated with gold nanoparticles and incorporated into liposomes (CGC-L). These preparations were incubated with HeLa cells for 3 hours at $37^{\circ} \mathrm{C}$.

TEM showed internalization of VGC-L into HeLa cells. As shown in Figure 4A, the gold nanoparticles (solid black dots) were distributed close to the nuclear membrane and in the lysosomes or endosomes. It was difficult to identify the organelle involved because it had a relatively small volume, similar to endosomes, and also possessed the electrondense characteristic of lysosomes. We determined that the 

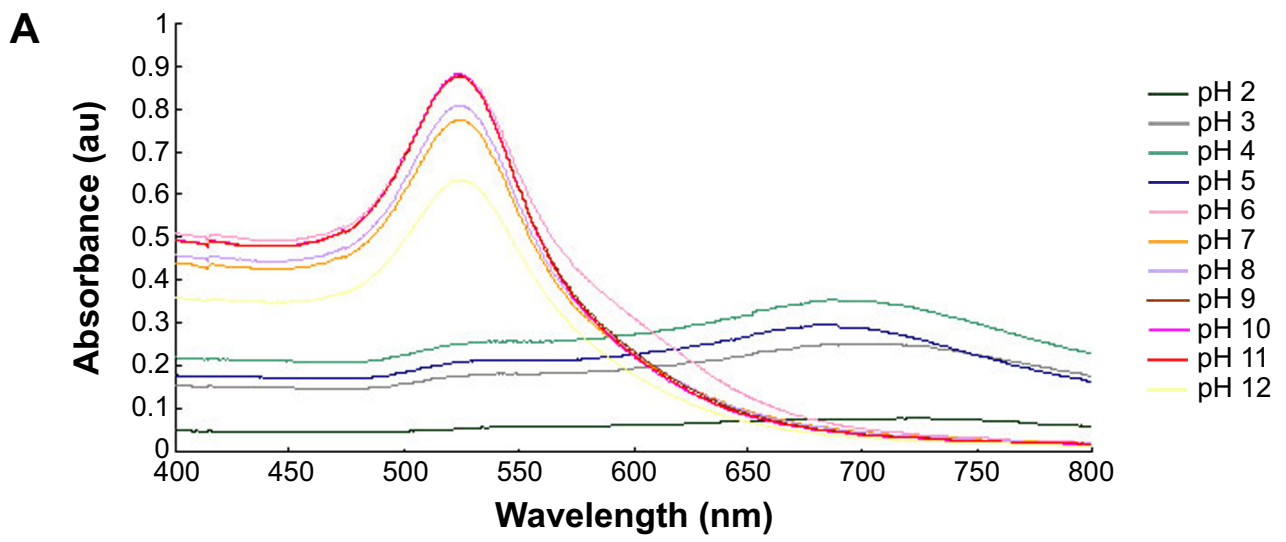

B

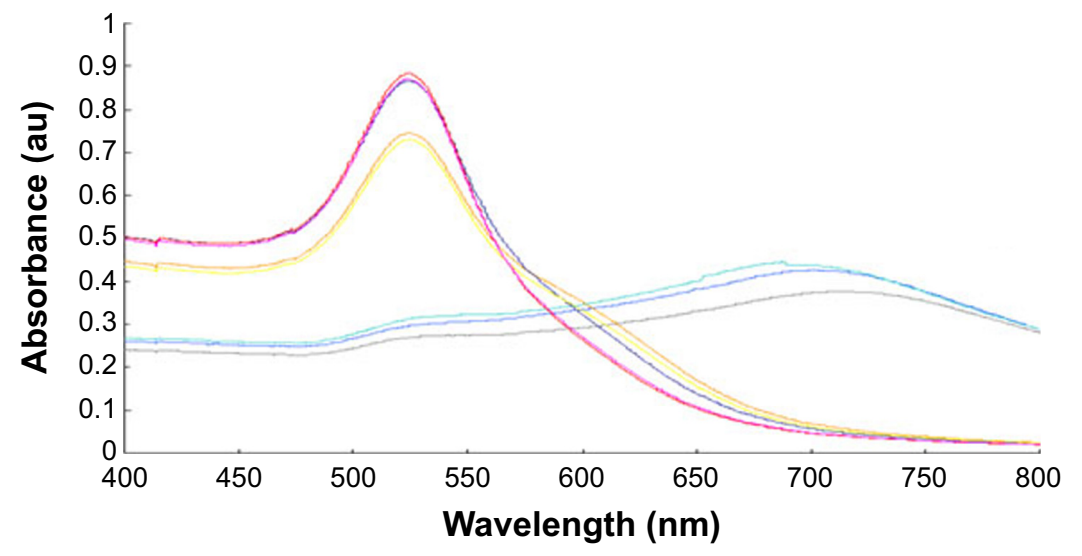

$-\mathrm{NaCl} 1$

$-\mathrm{NaCl} 10^{-1}$

- $\mathrm{NaCl} 10^{-2}$

$-\mathrm{NaCl} 10^{-3}$

$-\mathrm{NaCl} 10^{-4}$

$\mathrm{NaCl} 10^{-5}$

- $\mathrm{NaCl} 10^{-6}$

$-\mathrm{NaCl} 10^{-7}$
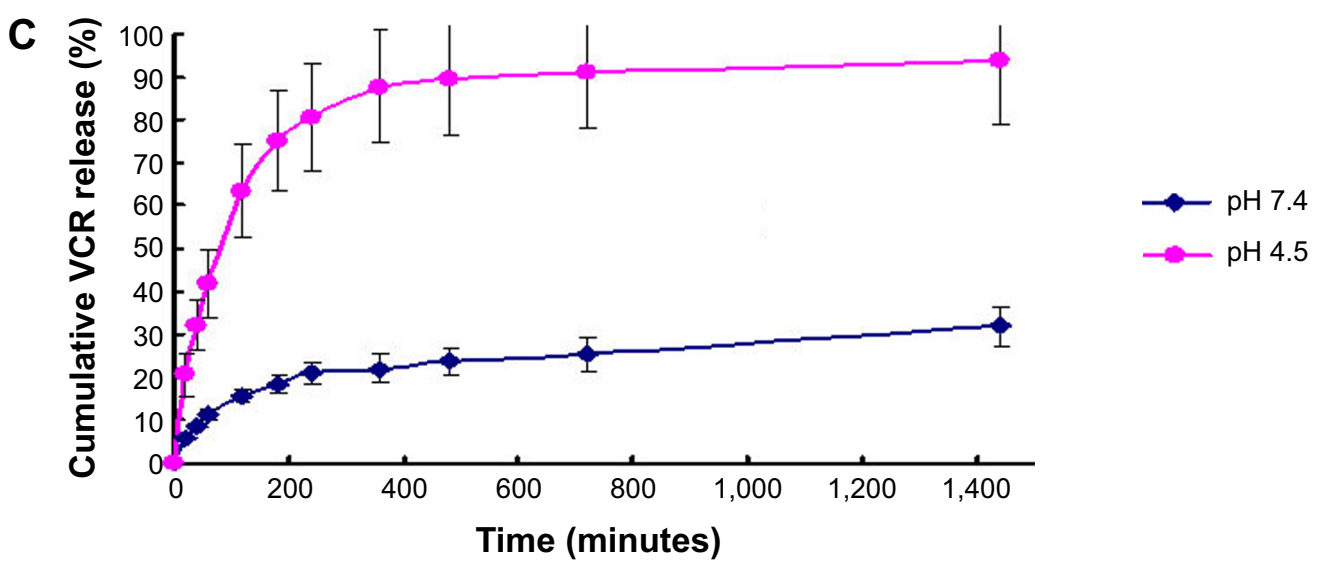

Figure 2 Characterization of VGC.

Notes: (A) UV/visible spectra of VGC at various $\mathrm{pH}$; (B) UV/visible spectra of VGC at various $\mathrm{NaCl}$ concentrations; (C) VCR release profile from VGC in phosphate buffer $(\mathrm{pH} 7.4)$ and acetate buffer $(\mathrm{pH} 4.5)$.

Abbreviations: VGC, vincristine sulfate-gold nanoparticles conjugates; VCR, vincristine sulfate.

compartment was most likely lysosomal, because liposomes are generally internalized by endocytosis into endosomes, followed by fusion with lysosomes. For the cells exposed to UV light, many more gold nanoparticles were localized to both endosomes (black dots numbered 2 and 3 ) and lysosomes (black dots numbered 4, 5, and 6).

Figure 5 shows the CLSM results obtained following nuclear and lysosomal staining. Weak green fluorescence was observed in cells treated with CGC-L for 0.5 hour, indicating that the liposomes were internalized by endocytosis. Merged images provided important information indicating that most of the liposomes were distributed near the nucleus (blue) and were accumulated in endosomes and lysosomes, as reflected by the overlap of red and green fluorescence. In addition, cellular uptake increased with time and higher fluorescent intensity was found in cells exposed to light at each time point. 

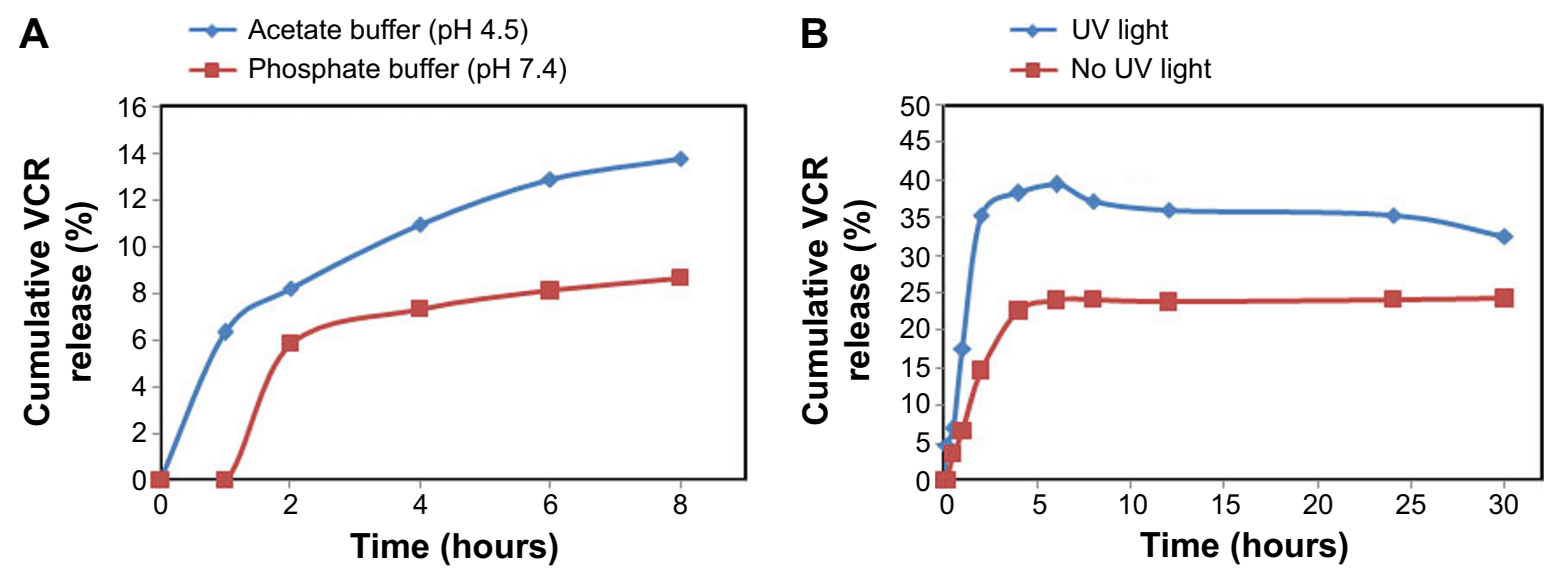

Figure 3 The drug release profile of VCR from VGC-L.

Notes: The drug release as measured in $(\mathbf{A})$ in phosphate buffer $(\mathrm{pH} 7.4)$ and acetate buffer $(\mathrm{pH} 4.5)$; (B) in acetate buffer ( $\mathrm{pH} 4.5)$ under 365 -nm $\mathrm{UV}$ light.

Abbreviation: VCR, vincristine sulfate; VGC-L, vincristine sulfate-gold nanoparticles conjugates loaded liposomes.

Flow cytometry was used to examine cellular uptake of CGC-L after 2 hours. As shown in Table 1, the highest fluorescent intensity was detected following light exposure, with less fluorescence observed in cells incubated with CGC-L without light $(P<0.01)$, and extremely low fluorescence in control cells. These findings confirmed that light enhanced the uptake of these liposomes.

\section{Enhanced cytotoxicity and apoptosis of HeLa cells exposed to light}

The effects of VGC-L on HeLa cell proliferation (with or without UV light) were determined in comparison to those of free VCR solution and VCR-L. As shown in Figure 4B, cells treated with VCR and VCR-L showed the same proliferation pattern irrespective of UV exposure, indicating that UV light did not influence their effects on cell viability. In contrast, significant enhancement of the effects of VGC-L on cell viability was observed as UV exposure times increased, indicating that UV light enhanced the antiproliferative effects of VGC-L.

The influence of VGC-L on cell apoptosis was evaluated using flow cytometry. HeLa cells that did not receive any treatment were used as the control. The cell apoptosis rates were $20.8 \%, 58.9 \%$, and $86.8 \%$ in cells exposed to VCR-L, VGC-L, and VGC-L with UV exposure, respectively. The higher cell apoptosis rate observed using VGC-L (as compared to VCR-L) may reflect the effects of both VCR and gold nanoparticles. The influence of VGC-L on HeLa cell apoptosis was also observed by optical microscopy (Figure S2). Cell morphology was normal, with closely packed cells, after 1-hour exposure to VCR-L. As the incubation time increased, spots of necrosis appeared. The morphology of cells exposed to VGC-L for 1 hour was altered, and a large number of cells were detached. In cells exposed to VGC-L under UV light, the cell bodies and nuclei were reduced in size.

\section{In vivo antitumor efficacy}

Treatment safety profiles were evaluated by measuring the body weights in each group. Compared with the negative control group, no significant differences in the body weights of nude mice treated with UV light, VGC-L, or VGC-L with UV exposure were observed (Figure 6A). This showed that UV light exposure had no associated toxicity. Mice treated with VGC-L (with or without UV light) were also in normal physical condition and no significant toxicity was observed. However, the body weight of the mice in the VCR group showed significant weight loss and signs of toxicity, with loss of appetite and reduced movement.

Figure $6 \mathrm{~B}$ shows the relative tumor volumes in nude mice, and Figure $6 \mathrm{C}$ shows the tumors after 16 days of treatment. Compared with the control group, the group exposed to UV light alone showed no difference in tumor size. There was a significant difference between the VGC-L group with UV exposure, and the negative control and UV light only groups. VGC-L with light exposure resulted in $74.1 \%$ tumor inhibition, which was significantly higher than the rates in the other three groups. No difference was observed between the VCR and VGC-L groups, with 59.5\% and 59.4\% tumor inhibition, respectively.

The extent of tumor cell apoptosis was examined in the isolated tumor sections 15 days postinjection, using the TUNEL assay. Figure 6D shows the dead cells (green), and the nucleus (blue). Only a small number of dead cells were observed in sections from control mice. The animals treated with VGC-L and UV-light showed a larger proportion of apoptotic tumor cells than those treated with VGC-L without light. 
A

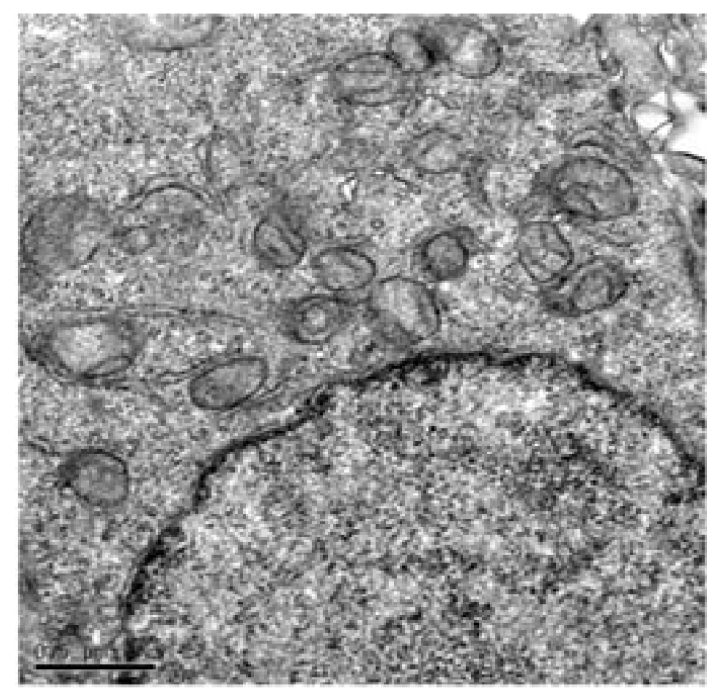

\section{VGC-L + UV}

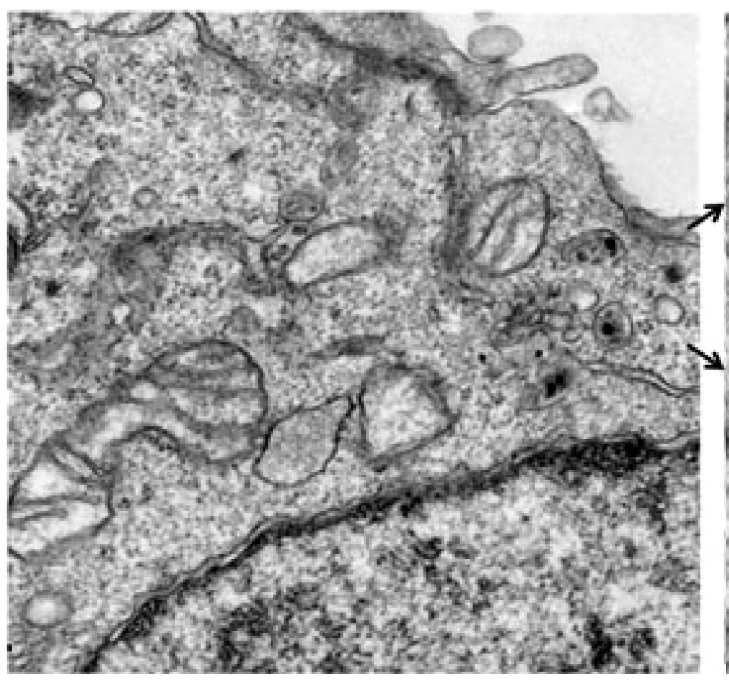

\section{VGC-L}
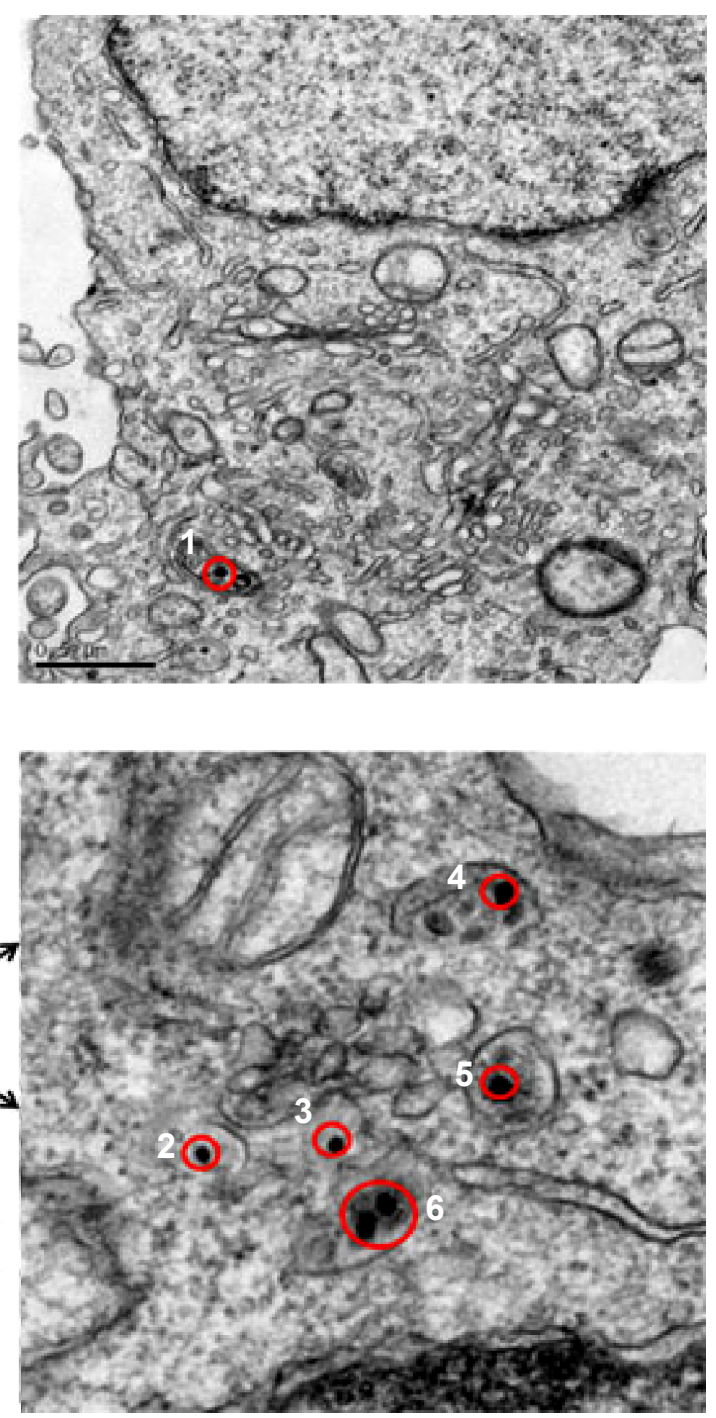

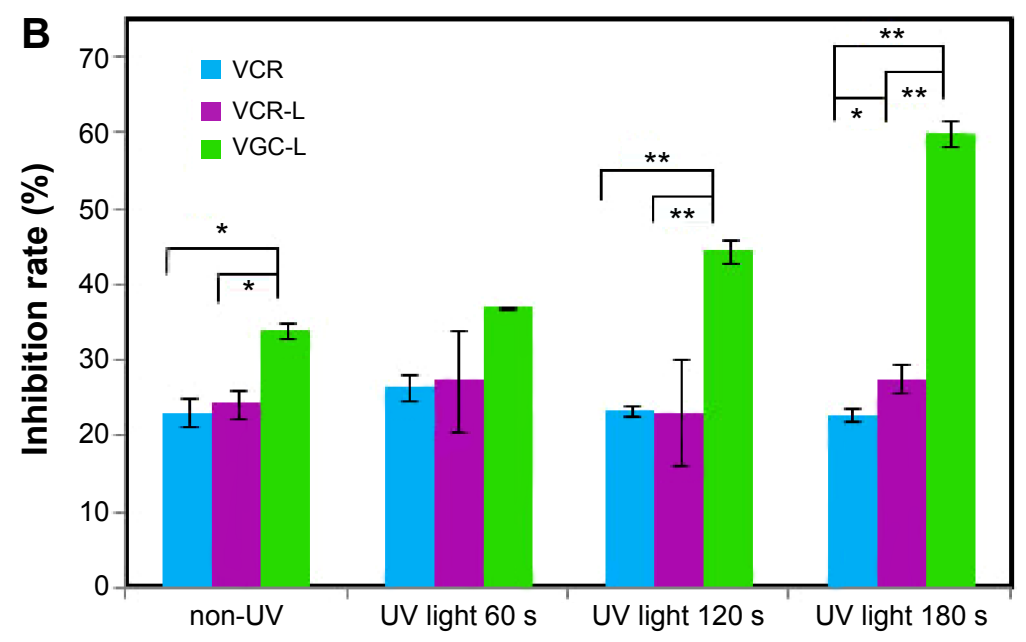

Figure 4 Cell uptake and cytotoxicity of VGC-L to Hela cells.

Notes: (A) TEM images of HeLa cells $(\times 30,000$ magnification). The red circles (I-6) represent the location of gold nanoparticles, the solid atrous dots in the circles are the gold nanoparticles, and the two arrows indicate the local area magnification; (B) The inhibition of HeLa cell viability following exposure to free VCR, VCR-L, and VGC-L with UV light for the indicated time periods.

Abbreviations: TEM, transmission electron microscopy; VCR, vincristine sulfate; VCR-L, vincristine sulfate-loaded liposomes; VGC-L, vincristine sulfate-gold nanoparticles conjugates loaded liposomes. 


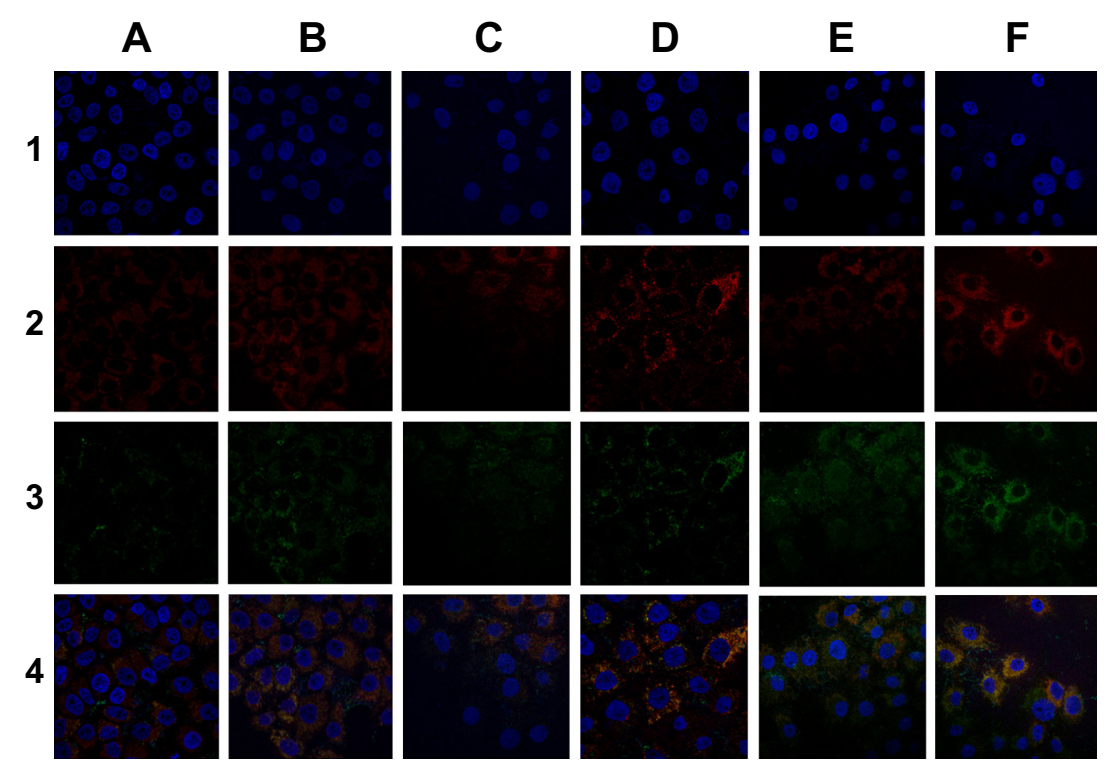

Figure 5 Confocal laser scanning microscopy images of HeLa cells treated for the indicated times with liposomes loaded with CGC-L or CGC-L plus UV light exposure. Notes: Column A, 0.5 h; B, 0.5 h + UV light; C, I h; D, I h + UV light; E, 2 h; F, 2 h + UV light. Row I, Hoechst 33258 (blue); 2, Lyso-Tracker Red (red); 3, calcein (green); 4, merged.

Abbreviation: CGC-L, calcein-conjugated gold nanoparticles loaded liposomes.

To determine the in vivo distribution of the prepared liposomes, in vivo fluorescence imaging was used following administration of RGC-L. ${ }^{32}$ As shown in Figure 7, RGC-L and UV light produced stronger fluorescence at the tumor site at each time point examined, as compared with mice not exposed to light. This may have indicated rapid UV-triggered release of rhodamine from RGC-L. Ex vivo imaging following the removal of tumors from nude mice at 32 hours postinjection confirmed that there was a greater fluorescent intensity in animals treated with RGC-L and UV light, than in those treated with RGC-L only. The RGC-L and UV laser-treated mice also showed stronger fluorescent intensity in the liver, kidney, and heart, as compared with the group not exposed to light. This result indicated that UV light triggered rhodamine release from RGC-L, followed by rhodamine absorption by the liver, and subsequent excretion via the kidneys.

\section{Discussion}

Liposomes are one of the most promising drug carriers for cancer treatment, both in preclinical development and clinical application, due to their good biological compatibility,

Table I Mean fluorescent intensity of HeLa cells exposed to liposomes loaded with calcein-conjugated gold nanoparticles (CGC-L) and CGC-L plus UV light, as determined by flow cytometry

\begin{tabular}{llll}
\hline & Control & CGC-L & CGC-L plus UV light \\
\hline Fluorescence intensity & $3.32 \pm 0.1$ & $39.04 \pm 1.47$ & $\mathrm{I} 15.87 \pm 3.66$ \\
\hline
\end{tabular}

reduced toxicity, and improved drug stability. To enhance the antitumor therapeutic efficacy of liposomes, stimuliresponsive liposomes are promising nanocarriers acting by controlling drug release. Therefore, in this study, gold nanoparticles-drug conjugates were incorporated into liposomes to investigate their antitumor efficiency. Particle size is an important issue in the design of gold nanoparticle drug delivery systems because it influences intracellular uptake, as well as toxicity. Gold nanoparticles with a particle size of 10-20 nm had good cellular uptake and reduced toxicity. ${ }^{33}$ The amount of trisodium citrate used in their manufacture influences gold nanoparticle size. ${ }^{34}$ The present study employed $1 \%$ trisodiumcitrate, which contains a carboxylic group as a capping and reducing agent, to synthesize gold nanoparticles. The yielded gold nanoparticles have shown desirable particle size and rapid cellular uptake.

The conjugation of gold nanoparticles with drug molecules may be achieved by various methods. ${ }^{35}$ In this study, VCR is an amphiphilic weak alkaline compound that is positively charged in aqueous dispersions, while the citrate-capped gold nanoparticles were negatively charged, as reflected by their zeta potential. This facilitated the formation of a physical complex between VCR and the gold nanoparticles via ionic bonding. Therefore, VGC were thus conveniently prepared by mixing VCR with the gold nanoparticles in an aqueous milieu. Studies using a combination of UV-visible spectroscopy, DSC, and FTIR, therefore, verified that VCR was grafted onto the gold nanoparticles. 

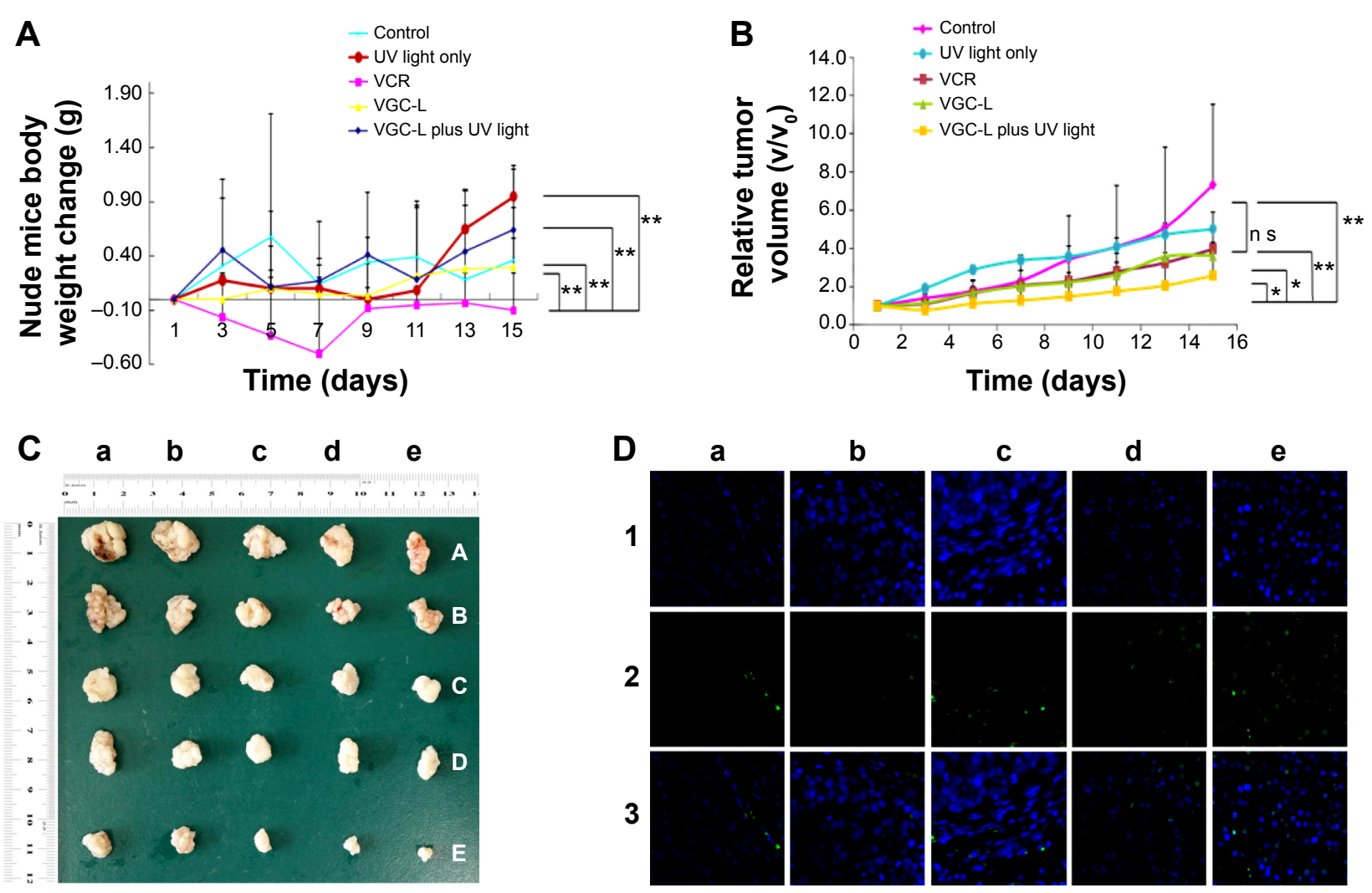

Figure 6 In vivo antitumor study in BALB/c mice implanted with HeLa cells.

Notes: (A) Body weight changes in control mice and those treated with UV laser only, VCR, VGC-L, and VGC-L plus UV laser ( $\mathrm{n}=5$ ). $* * P<0.0 \mathrm{I}$. (B) Relative tumor volume in control mice and those treated with UV laser only, VCR, VGC-L, and VGC-L plus UV laser $(n=5)$. $* P<0.05$, $* * P<0.01$. (C) Photographs of tumors after excision from (a) control; (b) only UV laser; (c) VCR; (d) VGC-L; and (e) VGC-L plus UV laser groups. (D) TUNEL staining of the isolated tumor tissue from (a) control; (b) blank plus UV laser; (c) VCR; (d) VGC-L; and (e) VGC-L plus UV laser groups. The apoptotic cells and cell nuclei were stained green and blue, respectively.

Abbreviation: VCR, vincristine sulfate; VGC-L, vincristine sulfate-gold nanoparticles conjugates loaded liposomes; TUNEL, Terminal-deoxynucleoitidyl Transferase Mediated Nick End Labeling.

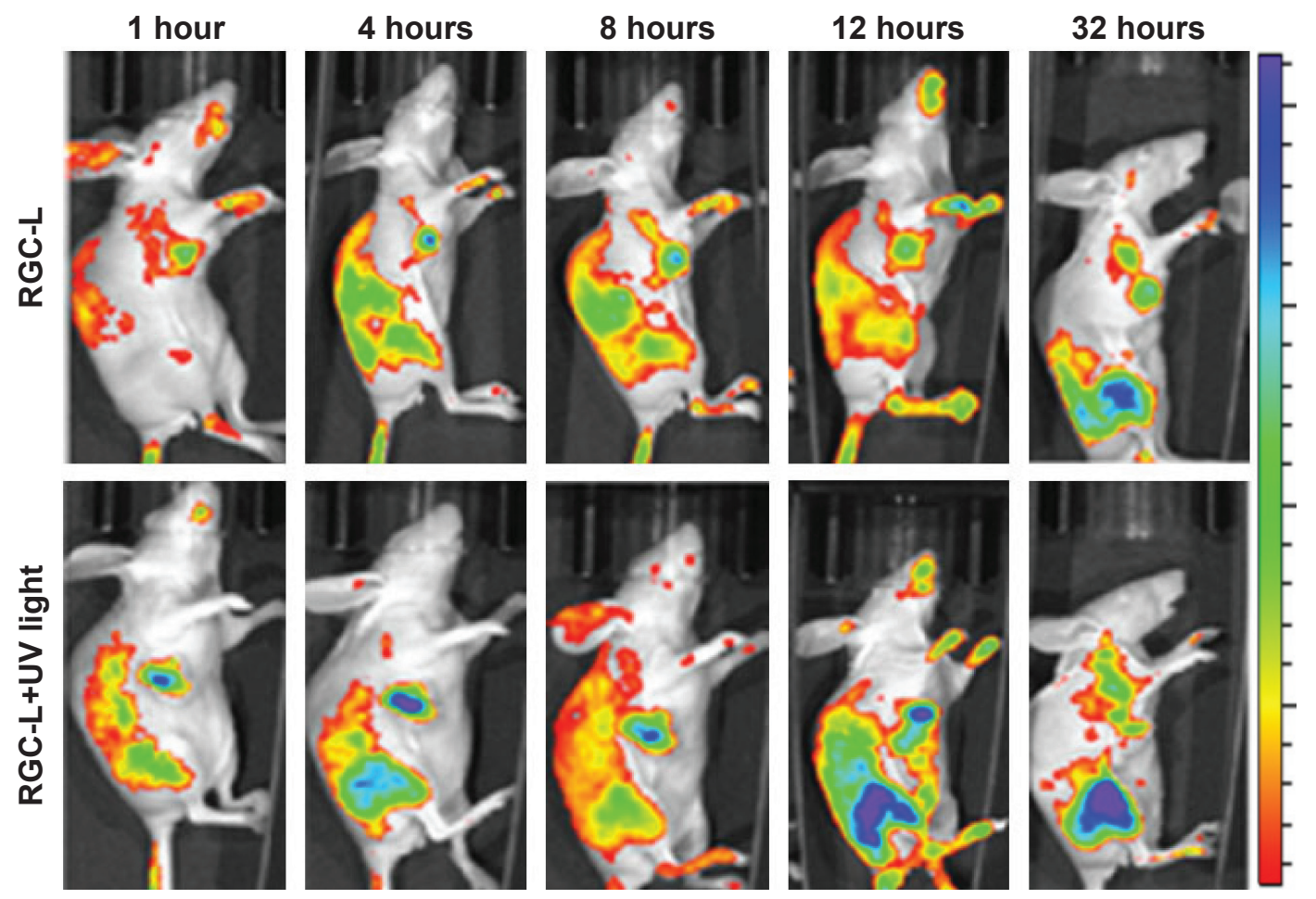

Figure 7 In vivo images of tumor-bearing nude mice treated with rhodamine B-conjugated gold nanoparticle-loaded liposomes (RGC-L) and RGC-L plus UV light. 
One strategy of this study is to utilize gold nanoparticles to achieve controlled and light-activated drug release, which is advantageous for antitumor therapy. As reflected in in vitro release study, the more rapid drug release under acidic conditions would be beneficial for site-specific drug release at tumors, given the pathophysiological nature of these tissues. ${ }^{36}$ This may reflect gold nanoparticle light absorption and conversion to thermal energy, creating a thermal phase transition in the lipid bilayers, improving permeability, and promoting release of the drug cargo. ${ }^{21,37,38}$

Intracellular uptake and distribution were studied to evaluate the intracellular delivery behavior of VGC-L, with or without light exposure. Combined the results of TEM, CLSM, and flow cytometry studies together, light enhanced cellular uptake of these liposomes into HeLa cells. Cellular uptake of nanocarriers has been shown to be influenced by many factors including size, charge, shape, encapsulated substance, and endocytosis pathway. ${ }^{39,40}$ In the present study, light stimulation accelerated drug release from VGC-L, as illustrated by the in vitro release study. This light-triggered release process may occur both intra- and extracellularly. The gold-drug conjugate had a small size of $\sim 50 \mathrm{~nm}$, indicating that if it was released extracellularly, it could be easily taken up by cells. ${ }^{20}$ In addition to intracellular drug release, extracellular release and subsequent rapid uptake by cells is also considered helpful in therapy. ${ }^{21}$ These TEM and CLSM results together indicated that internalized liposomes were mainly distributed to the endosomal-lysosomal compartment and that exposure to light improved their intracellular uptake.

Furthermore, the enhanced cytotoxicity and apoptosis of HeLa cells for VGC-L plus UV light irritation compared with VGC-L alone revealed that after gold nanoparticles as light-absorbing substances were taken up into tumor cells, the photothermal action upon light irritation contributed to stronger cell inhibition and improved tumor cells apoptosis. The phenomenon and mechanism of photothermal action have been illustrated in many studies; it was considered that heatinduced protein, DNA and/or RNA denaturation or unfolding may result in cytotoxicity and, therefore, apoptosis. ${ }^{41,42}$ Another factor contributing to the enhanced cytotoxicity was the light triggered VCR release from the VGC-L and increased intracellular free VCR concentration.

The enhanced antitumor efficiency for VGC-L plus UV light irritation compared with the liposomes alone, in vivo in the tumor bearing BALB/c nude mice, can be attributed to the following effects: 1) the enhanced permeability and retention effect of liposomes was expected to enhance
VGC-L accumulation in tumors, 2) light-triggered drug release could enhance the therapeutic effects of VCR and reduce its systemic toxicity, and 3) photothermal therapy mediated enhanced antitumor efficiency.

\section{Conclusion}

To improve the antitumor efficacy of VCR, VGC-L was developed in this study. It showed UV light-responsive release. In addition, light exposure enhanced intracellular drug release, cytotoxicity, and apoptosis in HeLa cells exposed to VGC-L. In vivo studies in tumor bearing nude mice confirmed that therapeutic efficacy was enhanced by light exposure, with a higher tumor inhibition rate and reduced side effects. Drug accumulation at the tumor site by the passive targeting effect of liposomes, together with light-responsive controlled release, produced greater and more rapid drug delivery to tumors and improved therapeutic efficacy. In conclusion, this drug delivery system provides a promising approach for antitumor applications.

\section{Acknowledgments}

This work was supported financially by the National Science Foundation of China(No 81195925, No 81283232) and Shanghai Natural Science Foundation Project (11ZR1416193).

\section{Disclosure}

The authors report no conflicts of interest in this work.

\section{References}

1. Cai D, Gao W, He B, et al. Hydrophobic penetrating peptide PFVYLImodified stealth liposomes for doxorubicin delivery in breast cancer therapy. Biomaterials. 2014;35:2283-2294.

2. Sampathkumar K, Arulkumar S, Ramalingam M. Advances in stimuli responsive nanobiomaterials for cancer therapy.J Biomed Nanotechnol. 2014; 10:367-382.

3. Thambi T, Deepagan VG, Yoon HY, et al. Hypoxia-responsive polymeric nanoparticles for tumor-targeted drug delivery. Biomaterials. 2014; 35:1735-1743.

4. Mo R, Sun Q, Li N, Zhang C. Intracellular delivery and antitumor effects of $\mathrm{pH}$-sensitive liposomes based on zwitterionic oligopeptide lipids. Biomaterials. 2013;34:2773-2786.

5. Connor EE, Mwamuka J, Gole A, Murphy CJ, Wyatt MD. Gold nanoparticles are taken up by human cells but do not cause acute cytotoxicity. Small. 2005;1:325-327.

6. Klassen NV, Kedrov VV, Ossipyan YA, et al. Nanoscintillators for microscopic diagnostics of biological and medical objects and medical therapy. IEEE Trans Nanobioscience. 2009;8:20-32.

7. Vigderman L, Zubarev ER. Therapeutic platforms based on gold nanoparticles and their covalent conjugates with drug molecules. Adv Drug Deliv Rev. 2013;65:663-676.

8. Iyer AK, Khaled G, Fang J, Maeda H. Exploiting the enhanced permeability and retention effect for tumor targeting. Drug Discov Today. 2006; 11:812-818.

9. Song M, Wang X, Li J, Zhang R, Chen B, Fu D. Effect of surface chemistry modification of functional gold nanoparticles on the drug accumulation of cancer cells. J Biomed Mater Res A. 2008;86:942-946. 
10. Jeong SY, Park SJ, Yoon SM, et al. Systemic delivery and preclinical evaluation of Au nanoparticle containing beta-lapachone for radiosensitization. J Control Release. 2009;139:239-245.

11. Polizzi MA, Stasko NA, Schoenfisch MH. Water-soluble nitric oxidereleasing gold nanoparticles. Langmuir. 2007;23:4938-4943.

12. Agasti SS, Chompoosor A, You CC, Ghosh P, Kim CK, Rotello VM. Photo regulated release of caged anticancer drugs from gold nanoparticles. J Am Chem Soc. 2009;131:5728-5729.

13. Mallidi S, Larson T, Aaron J, Sokolov K, Emelianov S. Molecular specific optoacoustic imaging with plasmonic nanoparticles. Opt Express. 2007;15:6583-6588.

14. Melancon MP, Lu W, Yang Z, et al. In vitro and in vivo targeting of hollow gold nanoshells directed at epidermal growth factor receptor for photothermal ablation therapy. Mol Cancer Ther. 2008;7:1730-1739.

15. Hainfeld JF, Slatkin DN, Smilowitz HM. The use of gold nanoparticles to enhance radiotherapy in mice. Phys Med Biol. 2004;49:N309-N315.

16. Lee J, Chatterjee DK, Lee MH, Krishnan S. Gold nanoparticles in breast cancer treatment: promise and potential pitfalls. Cancer Lett. 2014;347: 46-53.

17. Mady MM, Fathy MM, Youssef T, Khalil WM. Biophysical characterization of gold nanoparticles-loaded liposomes. Phys Med. 2012;28: 288-295.

18. Kim D, Park S, Lee JH, Jeong YY, Jon S. Antibiofouling polymercoated gold nanoparticles as a contrast agent for in vivo X-ray computed tomography imaging. J Am Chem Soc. 2008;129:7661-7665.

19. Kojima C, Hirano Y, Yuba E, Harada A, Kono K. Preparation and characterization of complexes of liposomes with gold nanoparticles. Colloids Surf B Biointerfaces. 2008;66:246-252.

20. Chithrani DB, Dunne M, Stewart J, Allen C, Jaffray DA. Cellular uptake and transport of gold nanoparticles incorporated in a liposomal carrier. Nanomedicine. 2010;6:161-169.

21. Paasonen L, Sipila T, Subrizi A, et al. Gold-embedded photosensitive liposomes for drug delivery: triggering mechanism and intracellular release. J Control Release. 2010;147:136-143.

22. Li D, Li G, Li P, et al. The enhancement of transfection efficiency of cationic liposomes by didodecyldimethylammonium bromide coated gold nanoparticles. Biomaterials. 2010;31:1850-1857.

23. Agarwal A, Mackey MA, El-Sayed MA, Bellamkonda RV. Remote triggered release of doxorubicin in tumors by synergistic application of thermosensitive liposomes and gold nanorods. ACS Nano. 2011;5: 4919-4926.

24. Von White G 2nd, Chen Y, Roder-Hanna J, Bothun GD, Kitchens CL. Structural and thermal analysis of lipid vesicles encapsulating hydrophobic gold nanoparticles. ACS Nano. 2012;6:4678-4685.

25. Kassem LA, Gamal El-Din MM, Yassin NA. Mechanismms of vincristine-induced neurotoxicity: Possible reversal by erythropoietin. Drug Discov Ther. 2011;5:136-143.

26. Zhang P, Ling G, Sun J, et al. Multifunctional nanoassemblies for vincristine sulfate delivery to overcome multidrug resistance by escaping P-glycoprotein mediated efflux. Biomaterials. 2011;32:5524-5533.

27. Storhoff JJ, Elghanian R, Mucic RC, Mirkin CA, Letsinger RL. One-pot colorimetric differentiation of polynucleotides with single base imperfections using gold nanoparticle probes. J Am Chem Soc. 1998;120: 1959-1964.
28. Zalba S, Navarro I, Troconiz IF, Tros de IIarduya C, Garrido MJ. Application of different methods to formulate PEG-liposomes of oxaliplatin: evaluation in vitro and in vivo. Eur J Pharm Biopharm. 2012; $81: 273-280$

29. Lazarus GG, Revaprassdu N, Lopez-Viota J, Singh M. The electrokinetic characterization of gold nanoparicles, functionalized with cationic functional groups, and its' interaction with DNA. Colloids Surf B Biointerfaces. 2014;121:425-431.

30. Anuradha J, AbbasiTasneem, Abbasi SA. An eco-friendly method of synthesizing gold nanoparticles using an otherwise worthless weed pistia (Pistiastratiotes L.). J Advanc Res. 2014; in press; doi: 10.1016/j. jare.2014.03.006.

31. Zhang X, Guo S, Fan R, et al. Dual-functional liposome for tumor targeting and overcoming multidrug resistance in hepatocellular carcinoma cells. Biomaterials. 2012;33:7103-7114.

32. Wang Y, Dou L, He H, Zhang Y, Shen Q. Multifunctional nanoparticles as nanocarrier for vincristine sulfate delivery to overcome tumor multidrug resistance. Mol Pharm. 2014;11:885-894.

33. Pan Y, Neuss S, Leifert A, et al. Size-dependent cytotoxicity of gold nanoparticles. Small. 2007;3:1941-1949.

34. Frens $\mathrm{G}$. Controlled nucleation for regulation of particle-size in monodisperse gold suspension. Nat Phys Sci. 1973;241:20-22.

35. Pissuwan D, Niidome T, Cortie MB. The forthcoming applications of gold nanoparticles in drug and gene delivery systems. J Control Release. 2011;149:65-71.

36. Danhier F, Feron O, Préat V. To exploit the tumor microenvironment: Passive and active tumor targeting of nanocarriers for anti-cancer drug delivery. J Control Release. 2010;148:135-146.

37. An X, Zhang F, Zhu Y, Shen W. Photo induced drug release from thermosensitive Gold nanoparticles-liposome using a Gold nanoparticlesswitch. Chem Commun (Camb). 2010;46:7202-7204.

38. An X, Zhan F, Zhu Y. Smart photothermal-triggered bilayer phase transition in Gold nanoparticles-liposomes to release drug. Langmuir. 2013;29:1061-1068

39. Xie S, Tao Y, Pan Y, et al. Biodegradable nanoparticles for intracellular delivery of antimicrobial agents. J Control Release. 2014;187: $101-117$.

40. Shahbazi MA, Almeida PV, Makila EM, et al. Augmented cellular trafficking and endosomal escape of porous silicon nanoparticles via zwitter ionic bilayer ploymer surface engineering. Biomaterials. 2014 35:7488-7500.

41. He XM, Wolkers WF, Crowe JH, Swanlund DJ, Bischof JC. In situ thermal denaturation of proteins in dunning AT-1 prostate cancer cells: implication for hyperthermic cell injury. Ann Biomed Eng. 2004;32: 1384-1398.

42. You J, Zhang R, Zhang G, et al. Photothermal-chemotherapy with doxorubicin-loaded hollow gold nanospheres: a platform for near-infrared light-trigged drug release. J Control Release. 2012;158:319-328. 


\section{Supplementary materials}

A

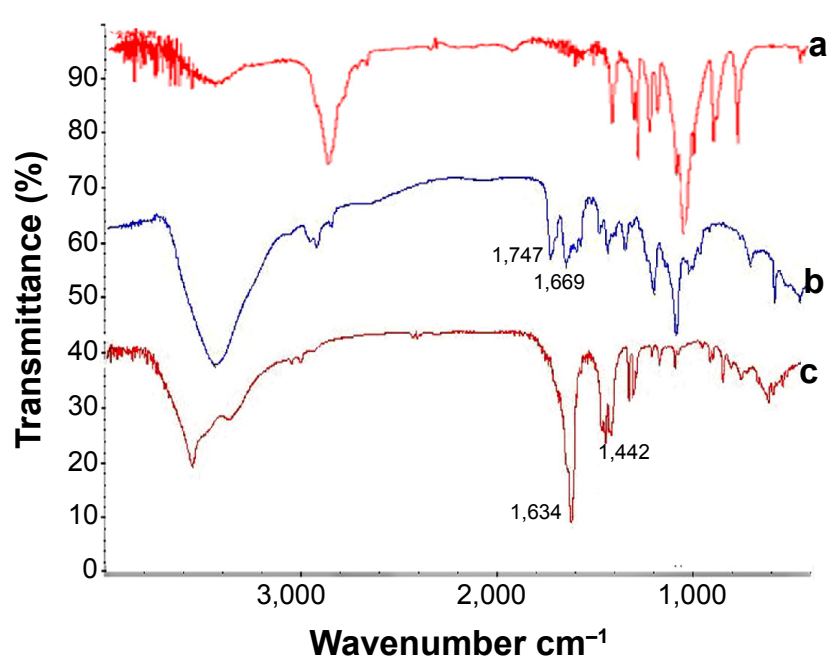

B

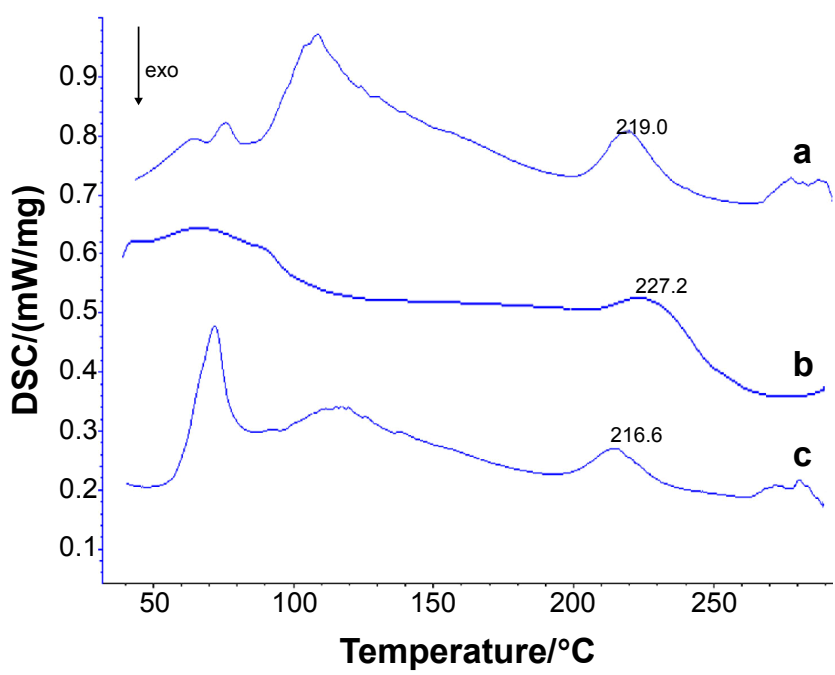

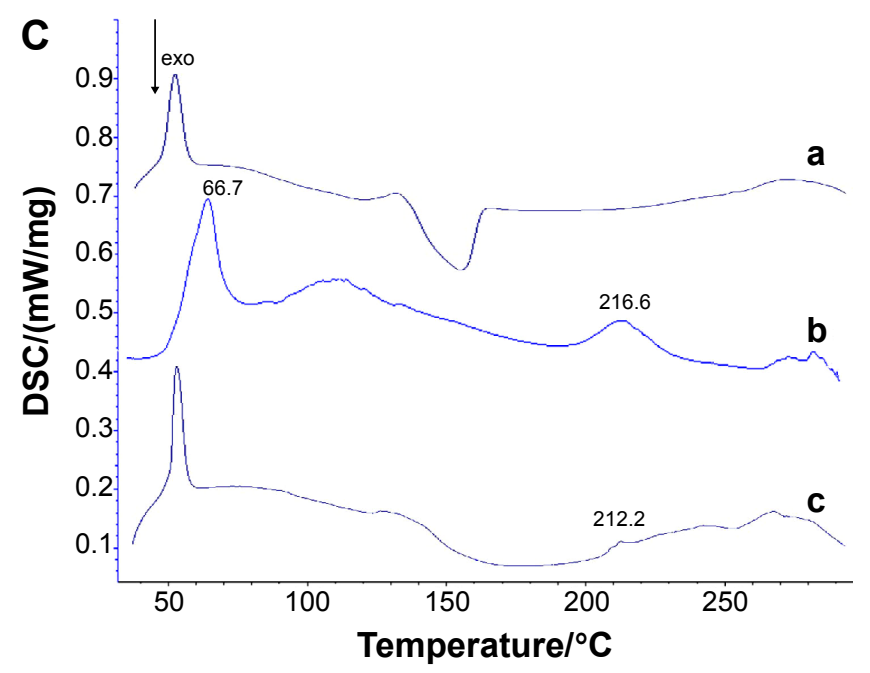

Figure SI (A) FTIR spectra of (a) gold nanoparticles; (b) VCR; and (c) VGC. (B) DSC curves of (a) gold nanoparticles; (b) VCR; and (c) VGC. (C) DSC curves of (a) BL; (b) VGC; (c) VGC-L.

Abbreviations: FTIR, fourier transform infrared spectroscopy; VCR, vincristine sulfate; VGC, vincristine sulfate-gold nanoparticles conjugates; DSC, differential scanning calorimetry; BL, blank liposomes; VGC-L, vincristine sulfate-gold nanoparticles conjugates loaded liposomes. 

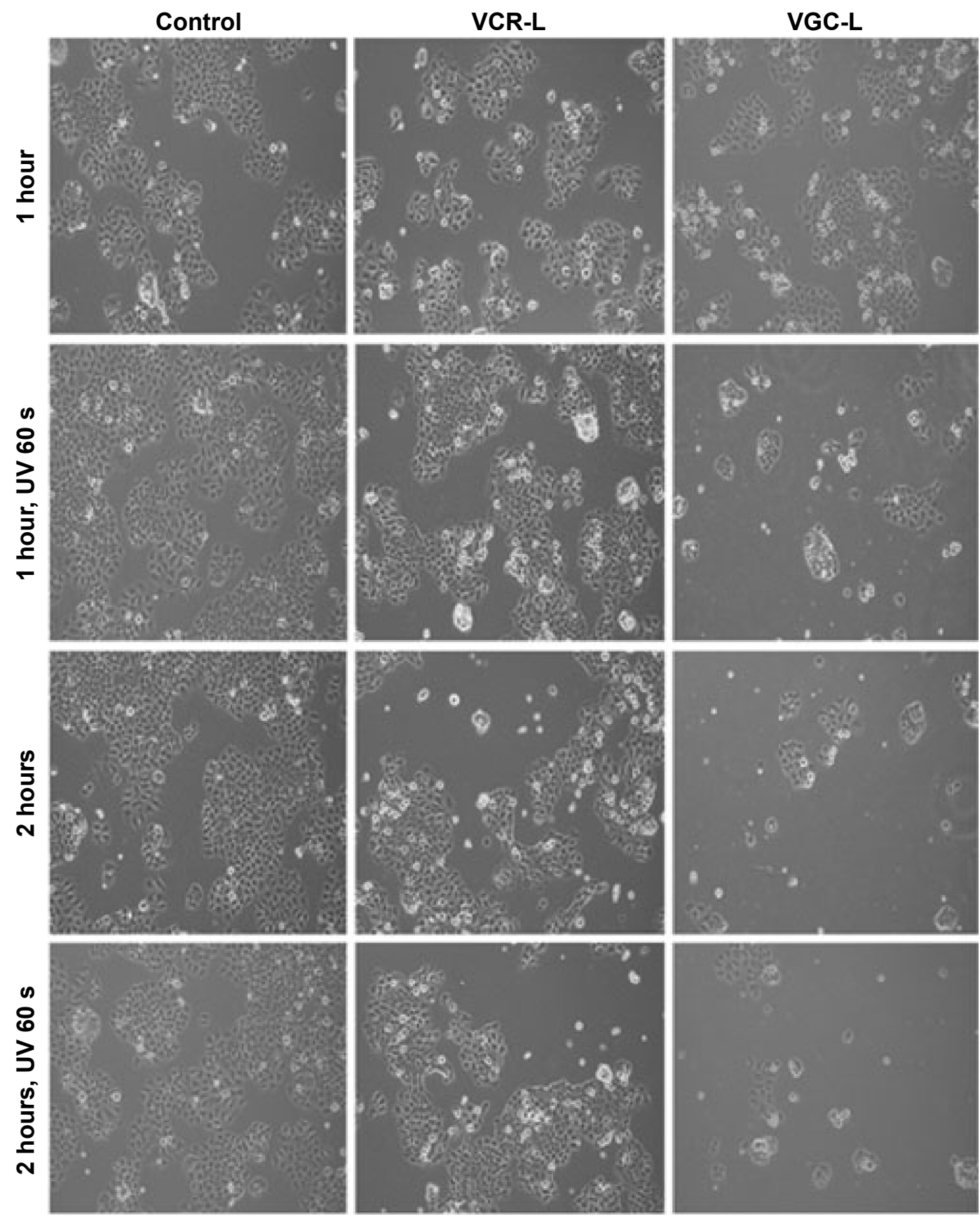

Figure S2 Microscope images of control HeLa cells and those incubated with VCR-L, VGC-L, and VGC-L plus UV light.

Abbreviations: VCR-L, vincristine sulfate-loaded liposomes; VGC-L, vincristine sulfate-gold nanoparticles conjugates loaded liposomes.

International Journal of Nanomedicine

\section{Publish your work in this journal}

The International Journal of Nanomedicine is an international, peerreviewed journal focusing on the application of nanotechnology in diagnostics, therapeutics, and drug delivery systems throughout the biomedical field. This journal is indexed on PubMed Central,

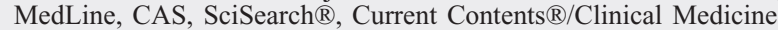

\section{Dovepress}

Submit your manuscript here: http://www.dovepress.com/international-journal-of-nanomedicine-journal 